\title{
ESTRUTURA DA COMUNIDADE ARBÓREA DA FLORESTA ATLÂNTICA DE BAIXADA PERIODICAMENTE INUNDADA Na RESERVA BIOLÓGICA DE Poço das Antas, Rio de Janeiro, Brasil
}

\author{
Fabrício Alvim Carvalho ${ }^{1,2}$, Marcelo Trindade Nascimento ${ }^{1}$, \\ João Marcelo Alvarenga Braga ${ }^{1,3}$ \& Pablo José Francisco Pena Rodrigues ${ }^{1,3}$
}

\begin{abstract}
Resumo
(Estrutura da comunidade arbórea da floresta atlântica de baixada periodicamente inundada na Reserva Biológica de Poço das Antas, Rio de Janeiro, Brasil) Este estudo teve por objetivo descrever a estrutura e composição florística arbórea de dois fragmentos naturais de florestas periodicamente inundadas na Reserva Biológica de Poço das Antas e compará-los a outros tipos florestais da região. Seis parcelas de $30 \mathrm{~m}$ x $20 \mathrm{~m}$ foram alocadas em cada fragmento. Todas as árvores (DAP $\geq 10 \mathrm{~cm}$ ) foram amostradas. Os dois fragmentos, denominados ARI e CM, apresentaram estrutura muito similar. Ao todo foram amostradas 628 árvores pertencentes a 31 espécies e 16 famílias, com forte dominância de espécies heliófilas tolerantes à inundação, como Calophyllum brasiliense, Symphonia globulifera (Clusiaceae) e Tabebuia cassinoides (Bignoniaceae). Estas três espécies foram responsáveis por 73\% do valor de cobertura em ARI e 67\% em CM. Os índices de diversidade de espécies ( $\mathrm{H}^{\prime}=1,75$ e 1,99, para ARI e CM respectivamente) foram próximos aos de outras florestas inundáveis do sudeste brasileiro. Os fragmentos apresentaram elevada similaridade florística devido à dominância destas espécies heliófilas. Entretanto, a similaridade de espécies foi baixa em relação às florestas bem drenadas adjacentes. Aparentemente, o alagamento do solo é o maior fator regulador da composição florística desta vegetação.
\end{abstract}

Palavras-chave: Floresta alagada, fragmentação, floresta atlântica de baixada, fitossociologia.

\section{Abstract}

(Tree community structure of a lowland seasonally flooded Atlantic Forest at Poço das Antas Biological Reserve, Rio de Janeiro, Brazil) This study aimed to describe the tree structure and floristic composition of two seasonally flooded forests at Poço das Antas Biological Reserve and to compare these forests to other Atlantic Forest types in the region. Six plots of $30 \mathrm{~m}$ x $20 \mathrm{~m}$ were set up in each fragment. All trees (DBH $\geq 10 \mathrm{~cm}$ ) were sampled. The fragments (ARI and CM) were structurally very similar. A total of 628 trees were sampled being distributed within 16 families and 31 species, with strong dominance of flooding tolerant heliophilous trees, such as Calophyllum brasiliense, Symphonia globulifera (Clusiaceae) and Tabebuia cassinoides (Bignoniaceae). This three species together accounted for $73 \%$ of cover value in ARI and $67 \%$ in CM. The species diversity $\left(\mathrm{H}^{\prime}=1,75\right.$ and 1,99 , respectively to ARI and $\left.\mathrm{CM}\right)$ can be considered to be within the values found for swamp forests in Southeastern Brazil. Both fragments showed high floristic similarity due to dominance of heliophilous trees. However, species similarity was low in comparison to adjacent well-drained Atlantic Forest. Soil flooding apparently was the major regulator factor of the floristic composition in this vegetation. Key words: Swamp forest, fragmentation, lowland Atlantic Forest, phytosociology.

\section{INTRODUÇÃo}

As terras úmidas, onde estão incluídos os brejos, pântanos, planícies de inundação e áreas similares, cobrem uma área estimada de $6 \%$ da superfície terrestre e estão entre os ecossistemas mais ameaçados do mundo (Maltby 1991). A América do Sul possui as maiores áreas de terras alagadas do mundo (Aselmann \& Crutzen 1989), e no território brasileiro, essas terras cobrem cerca de $2 \%$ da área (World Conservation Monitoring Centre 1992). No Brasil, as áreas alagadas são bastante extensas, sendo que as continentais recebem diversas designações, como banhado,

Artigo recebido em 10/2005. Aceito para publicação em 08/2006.

${ }^{1}$ Laboratório de Ciências Ambientais (LCA), Universidade Estadual do Norte Fluminense (UENF), Av. Alberto Lamego, 2000, Pq. Califórnia, 28015-620, Campos dos Goytacazes, RJ. mtn@uenf.br

${ }^{2}$ Programa de Pós-graduação em Ecologia, Instituto de Ciências Biológicas, Universidade de Brasília (UnB). Caixa Postal 04457, 70910-900, Brasília, DF. fabricioalvim@yahoo.com.br

${ }^{3}$ Instituto de Pesquisas Jardim Botânico do Rio de Janeiro (JBRJ). Rua Pacheco Leão 915, 22460-030, Rio de Janeiro, RJ. Apoio financeiro: MMA/PRONABIO/PROBIO/CNPq.jmabraga@jbrj.gov.br, pablo@jbrj.gov.br 
brejo, pântano, pantanal, igapó, várzea, campo úmido, lameiros e pampas (Esteves 1998). As maiores extensões de áreas alagadas encontram-se nas planícies de inundação de grandes rios, como a planície do rio Amazonas e seus afluentes, a do rio Paraguai (Pantanal) e a do rio Paraná, onde em geral o alagamento ocorre em ciclos anuais (Esteves 1998, Medri et al. 2002). As áreas alagadas associadas a rios de menores tamanhos são extensas e muito importantes ecologicamente, pois os períodos de inundação são mais curtos e os alagamentos podem não ocorrer anualmente (Medri et al. 2002). Estes ecossistemas são fundamentais para a manutenção dos recursos hídricos, da flora e da fauna local, sendo áreas de suprimento hídrico e alimentar para várias espécies da fauna (Maltby 1991, Naiman \& Décamps 1997) que em geral ocorrem como fragmentos naturais na Região Sudeste do Brasil (Constantino et al. 2005).

$\mathrm{O}$ alagamento interfere numa série de processos físico-químicos e biológicos, influenciando profundamente na qualidade do solo. Dentre as modificações destacam-se: diminuição de trocas gasosas entre o solo e o ar; lenta difusão do oxigênio; redução na biodisponibilidade de nutrientes; acúmulo de gases como N2, CO2 e H2 e aumento da produção de toxinas no solo (Medri et al. 2002). Uma vez que a limitação do oxigênio afeta o desenvolvimento e a sobrevivência das plantas superiores de forma diferenciada entre as espécies, a variação na sua disponibilidade consiste num forte determinante ecológico (Medri et al. 2002). Desta forma, o alagamento torna-se o maior condicionante da vegetação das comunidades arbóreas naturalmente inundáveis e sua influência é determinada pela freqüência, intensidade e duração das inundações, e relacionada localmente ao regime pluviométrico, relevo e tipo de solo (Joly 1991).

Os trechos de floresta atlântica inundável no sudeste brasileiro encontram-se, em geral, sobre solos hidromórficos e sujeitos à presença de água superficial em caráter temporário ou permanente, onde a saturação hídrica do solo é consequiência do afloramento da água do lençol freático (Leitão-Filho 1982, Ivanauskas et al. 1997, Toniato et al. 1998). No estado do Rio de Janeiro, esse tipo vegetacional encontra-se restrito a poucas áreas, restando cerca de $2 \%$ de sua área original (Câmara 1991). Estes fragmentos de florestas naturalmente ocorrem em manchas e apresentam grande relevância ecológica e conservacionista (Constantino et al. 2005), sendo consideradas áreas de preservação permanente pelo Código Florestal Brasileiro de 1965 (Milaré 1991).

Dentre os remanescentes de floresta atlântica de baixada do estado do Rio de Janeiro, destacam-se os da Reserva Biológica de Poço das Antas (IBDF 1981). No cenário de profunda fragmentação e degradação da floresta atlântica no Rio de Janeiro (Fundação SOS Mata Atlântica 2002) os remanescentes florestais desta Unidade de Conservação assumem importância fundamental como local de estudos e banco de germoplasma para futuros projetos de restauração ecológica (e.g. Programa Mata Atlântica 2004).

Mesmo considerando as recentes descrições florísticas (Scarano et al. 1997, 1998, Araujo et al. 1998), as florestas inundáveis do estado do Rio de Janeiro vêm recebendo pouca atenção da comunidade científica, ao contrário de outras vegetações inundáveis brasileiras, como as florestas de galeria do Brasil Central (Joly \& Crawford 1982, Oliveira-Filho 1989, Felfili 1993, Ratter et al. 1998), o pantanal mato-grossense (Prance \& Schaller 1982, Nascimento \& Cunha 1989, Loureiro et al. 1994, Ratter et al. 1998), as várzeas e igapós amazônicos (Prance 1979, Worbes 1985, Junk 1989, Kubitzky 1989, Schluter et al. 1993, Scarano et al. 1994), as florestas higrófilas de São Paulo (Leitão-Filho 1982, Joly 1991, Torres et al. 1994, Ivanauskas et al. 1997, Toniato et al. 1998) e as florestas ciliares do Paraná (Medri et al. 2002), cujos padrões florísticos e estruturais são bem conhecidos. Esta escassez de informações aliada à acelerada destruição deste habitat ressalta a importância de estudos sobre a estrutura e composição de suas comunidades, conforme proposto neste estudo. 


\section{Material e Métodos}

\section{Área de estudo}

A Reserva Biológica de Poço das Antas (Rebio) localiza-se na bacia hidrográfica do rio São João, município de Silva Jardim, RJ, Brasil (223'ㅇ's e $\left.42^{\circ} 15^{\prime} \mathrm{W}\right)$ (Fig. 1). Segundo a classificação de Veloso et al. (1991), esta região está inserida no domínio da Floresta Ombrófila Densa de Terras Baixas. A região pertence à Unidade Geomorfológica Colinas e Maciços Costeiros, uma área de relevo deprimido, com reduzidos valores altimétricos, situada em uma extensa planície de domínio quaternário (RADAMBRASIL 1983). Seu relevo é predominantemente de planície costeira, apresentando pequenos morros e morrotes (mamelonares) arredondados que chegam a atingir $200 \mathrm{~m}$ de altitude, imersos em áreas de baixadas aluviais sujeitas ao alagamento permanente ou periódico do solo, em função do regime pluvial (IBDF 1981, Takizawa 1995).

A Rebio possui uma área de aproximadamente 5.500 ha, dentre os quais cerca de 1.500 encontram-se em estado de inundação periódica, constituindo uma amostra do que restou desse tipo de habitat que foi drenado por vários canais projetados pelo Departamento Nacional de Obras e Saneamento (DNOS) para retificação dos rios e para o escoamento mais rápido das águas da região (Castro \& Fernandez 2002). Sua hidrologia compreende a bacia hidrográfica do rio São João, que abrange cerca de $2.080 \mathrm{~km}^{2}$. O rio São João é o principal curso de água diretamente relacionado com a Rebio, constituindo seu limite a sudoeste e sendo receptor de todas as águas que passam ou se originam na região. Na estação chuvosa o volume de águas do rio aumenta consideravelmente, inundando as terras baixas de seu vale (Moraes 2000).

O clima regional é do tipo megatérmico úmido (B2A') na classificação de Thornthwaite \& Mather, correspondendo à designação de tropical chuvoso com estação seca no inverno (As) de Köeppen (Bernardes 1952). O diagrama ombrotérmico referente ao período de janeiro de 1993 a dezembro de 2002 (Fig. 4) assinala os meses de junho a agosto como representantes do período menos chuvoso e de temperaturas menos elevadas, correspondendo os meses de novembro a março ao período mais chuvoso e de maiores temperaturas. Os demais meses representam situações intermediárias entre os dois períodos. Neste intervalo de dez anos, a temperatura média anual foi de $25,5^{\circ} \mathrm{C}$, sendo registrada no mês de julho a temperatura média mais baixa de $21,8^{\circ} \mathrm{C}$ e a maior temperatura média durante os meses de janeiro e fevereiro $\left(29^{\circ} \mathrm{C}\right)$. Em relação à precipitação,

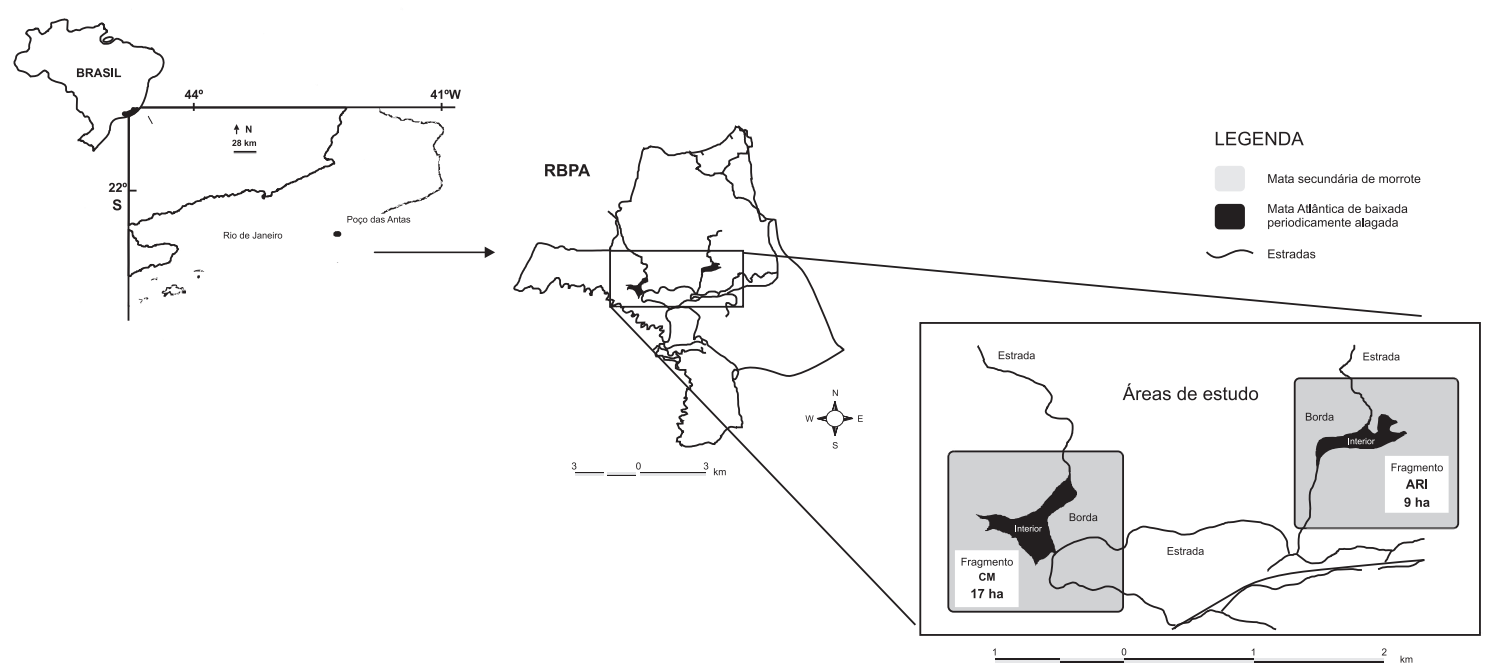

Figura 1 - Localização dos dois fragmentos de floresta de baixada periodicamente inundada, "brejo do Aristides" (ARI) e "brejo Casa dos Morcegos" (CM) na Reserva Biológica de Poço das Antas, Rio de Janeiro. 
foi observado no período um valor médio anual de 1994,9 mm, correspondendo ao mês de dezembro o maior índice (320 mm) e o menor valor $(38,9 \mathrm{~mm})$ ao mês de julho (Lima et al. 2006). Os solos encontrados nessas áreas são classificados como Gleissolos, aluviais e orgânicos (Takizawa 1995).

Em estudo fisionômico-florístico e de mapeamento da vegetação da Rebio, Lima et al. (2006) identificaram seis unidades fisionômicas, sendo duas florestais (floresta aluvial e floresta submontana) e quatro não florestais (formação pioneira com influência fluvial, capoeira aluvial, capoeira submontana e campo antrópico) e estimaram que dos 2.608 ha de remanescentes florestais ca.18\% (895 ha) eram de floresta aluvial.

Dentro da Rebio foram selecionados dois fragmentos naturais de floresta de baixada periodicamente alagada, circundados por florestas secundárias de morrote com até $50 \mathrm{~m}$ de altitude e distanciados um do outro cerca de $2 \mathrm{~km}$ (Fig. 1). Nestes enclaves encontram-se trechos com florestas de baixadas denominados "Brejo do Aristides" (ARI), com cerca de 9 ha, e "Brejo Casa dos Morcegos" (CM), com 17 ha. Ambas as áreas são baixadas aluviais de drenagem, cujos os solos ficam alagados no período das chuvas, entre os meses de outubro e abril (IBDF 1981). O solo apresenta micro-relevo irregular onde as plantas se distribuem sobre pequenos montes de até $50 \mathrm{~cm}$ de elevação, que constituem as partes emersas e mais altas do estrato inferior da floresta, formando pequenas "ilhas" de vegetação. O nível de água superficial normalmente não ultrapassa os $30 \mathrm{~cm}$ (Scarano et al. 1998), sendo comum a presença de canais de drenagem entre os "murundus". Aanálise da variação anual da coluna d'água nos fragmentos, realizada por D.M. Villela \& G.M. Gonçalves (dados não publicados), indicou para o ano de 2002 uma pequena variação na altura da coluna de água de $0,3 \mathrm{~cm}$ a $23,0 \mathrm{~cm}$ no fragmento ARI, visivelmente mais seco, e de 1,2 $\mathrm{cm}$ a 44,0 cm no fragmento CM entre os meses de janeiro a abril, e que entre abril e outubro os solos se encontraram totalmente secos. Há cerca de 35 anos esses fragmentos foram submetidos à extração seletiva de madeira, principalmente de "pau-tamanco" (Tabebuia cassinoides), e mudanças no regime hídrico, devido a obras de drenagem e construção de canais e represas na bacia do rio São João (Scarano et al. 1998, Castro \& Fernandez 2002). Não existem registros de perturbações antrópicas nesses fragmentos desde 1981, quando a área foi transformada em Reserva Biológica (IBDF 1981).

\section{Coleta e análise de dados}

Em cada fragmento foram estabelecidas duas áreas de $200 \mathrm{~m} \times 100 \mathrm{~m}$ (2,0 ha), uma em sua parte mais marginal distando, pelo menos, 20 $\mathrm{m}$ da borda, e outra em seu interior. Dentro de cada área foram alocadas aleatoriamente três parcelas de $30 \mathrm{~m} \times 20 \mathrm{~m}\left(600 \mathrm{~m}^{2}\right)$ para obter uma maior heterogeneidade florística uma vez que análises qualitativas prévias indicaram a ocorrência de densas populações de Tabebuia cassinoides, Calophyllum brasiliense e Symphonia globulifera. Ao todo foram alocadas seis parcelas por fragmento, totalizando uma área amostral de 0,36 ha. Em cada parcela, todas as árvores vivas e mortas em pé com DAP (diâmetro à altura do peito, a 1,30 $\mathrm{m}$ do solo) $\geq$ $10 \mathrm{~cm}$ foram marcadas, coletadas e medidas quanto à altura e ao DAP. Indivíduos perfilhados só foram marcados e medidos quando pelo menos um dos ramos possuía DAP $\geq 10 \mathrm{~cm}$, e nesse caso, todos os ramos foram medidos e a área basal total do indivíduo foi calculada pelo somatório das áreas basais de cada ramo. Informações tais como presença de resinas, látex, tipo de casca, cor e aroma do tronco e nome popular foram anotadas para o auxílio no trabalho de identificação das espécies. As amostras coletadas foram identificadas por comparação com as coleções do herbário do Jardim Botânico do Rio de Janeiro (RB) e do Herbário do Centro de Biociências e Biotecnologia da Universidade Estadual do Norte Fluminense (UENF) onde foi depositado todo o material botânico coletado. $\mathrm{O}$ sistema de classificação de famílias utilizado foi o de Cronquist (1981), exceto para Leguminosae que seguiu Polhill \& Raven (1981).

Os parâmetros fitossociológicos foram calculados através do programa FITOPAC 1 (Shepherd 1994), a saber: área basal total e 
individual (Abt e Abi), densidade relativa (DR), dominância relativa (DoR) e valor de cobertura (VC=DR+DoR) (Mueller-Dumbois \& Ellenberg 1974). O índice de uniformidade (J), o índice de diversidade de Shannon (H'), calculado utilizandose logaritmo natural, e o índice de similaridade de Morisita-Horn, para comparação florística entre os fragmentos estudados foram calculados segundo Brower \& Zar (1984). O índice de similaridade de Sørensen (Brower \& Zar 1984) foi utilizado para a comparação com outras áreas florestais brasileiras.

A suficiência amostral foi avaliada através do cálculo de curvas de rarefação, com 1000 iterações, utilizando os valores de riqueza e de diversidade de espécies $\left(\mathrm{H}^{\prime}\right)$ através do programa Ecosim (Gotelli \& Entsminger 2001).

As espécies levantadas foram classificadas segundo sua preferência por áreas alagadas, como proposto por Ivanauskas et al. (1997), em quatro grupos distintos: (1) Espécies peculiares exclusivas de formações brejosas de ocorrência exclusiva a florestas sujeitas ao alagamento permanente ou quase permanente do solo, possuem área de ocorrência geográfica restrita, e seu destaque na estrutura da comunidade está diretamente ligado a um maior período de saturação hídrica do solo; (2) Espécies peculiares não exclusivas de formações brejosas - destacam-se em florestas sujeitas ao alagamento periódico do solo, mas nunca em áreas com alagamento permanente ou quase permanente do solo ou em outras florestas com solos mais drenados, sendo comum sua ocorrência em florestas de galeria, ciliares e ripárias; (3) Espécies complementares de áreas secas - destacam-se em florestas com alagamento periódico do solo (florestas de galeria e ripárias) ou em florestas mais secas, mas nunca em florestas com alagamento permanente ou quase permanente do solo; e (4) Espécies complementares indiferentes-ocorrem em áreas alagadas, contudo sua abundância e densidade não têm relação direta com o alagamento. Para tal classificação, foram obtidas informações ecológicas sobre as espécies por meio de literatura especializada e consultas a levantamentos florísticos e fitossociológicos realizados em diferentes trechos de floresta atlântica do sudeste brasileiro. Algumas espécies não puderam ser classificadas devido à falta de informações ecológicas sobre as mesmas.

\section{Resultados}

Os dois fragmentos analisados (ARI e CM) apresentaram composições florísticas muito semelhantes, confirmada pelo alto valor encontrado para o índice de similaridade de Morisita-Horn $(0,83)$. Dentre as espécies que caracterizam o dossel desta formação, destacaram-se como as mais abundantes Calophyllum brasiliense, Symphonia globulifera, Tabebuia cassinoides, Henriettea saldanhaei, Myrcia multiflora, Platymiscium floribundum, Inga laurina e Tabebuia umbellata (Tab. 1). A altura média do dossel variou entre $12 \mathrm{~m} \mathrm{e} 15 \mathrm{~m}$, com alguns indivíduos emergentes de Calophyllum brasiliense, Platymiscium floribundum, Symphonia globulifera e Tapirira guianensis atingindo de $20 \mathrm{~m}$ a 25 m. Em relação à área basal por hectare, os valores encontrados foram também muito semelhantes entre os fragmentos $\left(36,5 \mathrm{~m}^{2} \mathrm{em}\right.$ ARI e $37,0 \mathrm{~m}^{2}$ em CM).

No total foram amostrados 628 indivíduos (361 em ARI e 267 em CM) distribuídos em 16 famílias, 23 gêneros e 31 espécies, dentre as quais uma foi identificada apenas em nível de família (Polygonaceae sp.) (Tab. 1). A suficiência amostral foi confirmada pela assíntota das curvas de rarefação calculadas, tanto para a riqueza quanto para a diversidade de espécies ( $\left.\mathrm{H}^{\prime}\right)$, para cada um dos fragmentos estudados (Fig. 2). A maioria das famílias apresentou baixa riqueza de espécies, com uma ou duas espécies apenas. As únicas famílias de destaque na riqueza foram Myrtaceae, com sete espécies, e Leguminosae Mimosoideae, com cinco. Clusiaceae foi a família com maior VC nos dois fragmentos estudados, devido a elevada densidade de Symphonia globulifera e principalmente Calophyllum brasiliense, espécie de maior VC em ambas as áreas. Bignoniaceae apareceu como a segunda família mais importante nos fragmentos, devido à elevada densidade de Tabebuia cassinoides. Estas três espécies foram as mais representativas 
Tabela 1 - Listagem de famílias e espécies arbóreas, com nome vulgar, número de indivíduos e ocorrência nos dois fragmentos de floresta de baixada periodicamente inundada (ARI e CM) estudados na Reserva Biológica de Poço das Antas, RJ. PA = preferência por áreas alagadas $(\mathrm{Pe}=$ espécies peculiares exclusivas de áreas alagadas; $\mathrm{Pn}=$ peculiares não exclusivas de áreas alagadas; $\mathrm{Cs}=$ complementares de áreas secas; $\mathrm{Ci}$ = complementares indiferentes; $\mathrm{Nc}=$ não classificada).

\begin{tabular}{|c|c|c|}
\hline Família & Nome vulgar & $\mathbf{N}^{o}$ indivíduos \\
\hline Espécie & & ARI CM \\
\hline
\end{tabular}

ANACARDIACEAE

Tapirira guianensis Aubl.

ARECACEAE

Euterpe edulis Mart.

BIGNONIACEAE

Tabebuia cassinoides (Lam.) DC.

Tabebuia umbellata (Sond.) Sandwith

\section{BOMBACACEAE}

Eriotheca pentaphylla (Vell. emend.

K. Schum.) A. Robyns

Pseudobombax grandiflorum (Cav.) A. Robyns imbiruçu

BORAGINACEAE

Cordia sellowiana Cham.

Calophyllum brasiliense Cambess.

Symphonia globulifera L. f.

\section{EUPHORBIACEAE}

Alchornea triplinervia (Spreng) Müll. Arg.

\section{LEGUMINOSAEMIMOSOIDEAE.}

Inga edulis Mart.

Inga laurina (Sw.) Willd.

Inga striata Benth.

Mimosa bimucronata (DC.) Kuntze

Pseudopiptadenia contorta (DC.)

G. P.Lewis \& M. P. Lima

LEGUMINOSAE PAPILIONOIDEAE.

Platymiscium floribundum Vog.

Andira anthelmia (Vell.) J. F. Macbr.

MAGNOLIACEAE

Talauma ovata A. St.-Hil.

MELASTOMATACEAE

Henriettea saldanhaei Cogn.

Tibouchina granulosa (Desr.) Cogn. chá-de-bugre

guanandi

guanandi

fruto-do-pombo

palmito, juçara

ipê-tamanco, caixeta

ipê-do-brejo

imbiruçu-branco

pau-tamanco, tapiá

ingá-cipó

ingá-mirim

Ingá

espinhenta, maricá

angico

jacarandá, sacambu

angelim, morcegueira

pinheiro-do-brejo

pixiricão-do-brejo

quaresmeira
Pn

$\begin{array}{lll}\mathrm{Ci} & - & 1\end{array}$

Pn $\quad$ - 16

Cs $\quad-1$

Cs 2 -

Cs $\quad-1$

Pe

$2-$

$\begin{array}{lll}\mathrm{Pe} & 40 & 52\end{array}$

$\begin{array}{lll}\mathrm{Pe} & 3 & 16\end{array}$

G

$54 \quad 40$

$\begin{array}{lll}\text { Pn } & 34 & - \\ \text { Cs } & 1 & -\end{array}$

Nc $\quad-\quad 1$




\begin{tabular}{|c|c|c|c|c|}
\hline \multirow{2}{*}{$\begin{array}{l}\text { Família } \\
\text { Espécie }\end{array}$} & \multirow[t]{2}{*}{ Nome vulgar } & \multirow[t]{2}{*}{ PA } & \multicolumn{2}{|c|}{$\mathrm{N}^{\circ}$ indivíduos } \\
\hline & & & ARI & $\mathbf{C M}$ \\
\hline \multicolumn{5}{|l|}{ MYRSINACEAE } \\
\hline Myrsine coriacea (Sw.) R. Br. ex Roem. \& Schult. & - & Cs & 1 & - \\
\hline \multicolumn{5}{|l|}{ MYRTACEAE } \\
\hline Calyptranthes brasiliensis Spreng. & - & Cs & - & 1 \\
\hline Gomidesia fenzliana $\mathrm{O}$. Berg & - & Cs & - & 2 \\
\hline Marlierea silvatica Kiaersk. & - & $\mathrm{Nc}$ & 1 & - \\
\hline Myrcia multiflora (Lam.) DC. & - & Cs & - & 37 \\
\hline Myrcia hexasticha Kiaersk. & - & $\mathrm{Nc}$ & - & 1 \\
\hline Myrcia racemosa (O. Berg) Kiaersk. & - & Pn & - & 2 \\
\hline Syzygium jambos (L.) Alston & jambo branco & $\mathrm{Ci}$ & 2 & 1 \\
\hline \multicolumn{5}{|l|}{ NYCTAGINACEAE } \\
\hline Guapira hoehnei (Standl.) Lundell & maria-mole & Cs & 3 & - \\
\hline \multicolumn{5}{|l|}{ POLYGONACEAE } \\
\hline Polygonaceae sp.1 & - & $\mathrm{Nc}$ & 2 & 4 \\
\hline \multicolumn{5}{|l|}{ SAPOTACEAE } \\
\hline Sarcaulus brasiliensis (A. DC.) Eyma & - & Pn & 1 & - \\
\hline
\end{tabular}

em ambos os fragmentos (Tabs. 2 e 3), e em conjunto corresponderam a 71,6\% do número de indivíduos amostrados e $70,5 \%$ do VC total. Dentre as espécies restantes, algumas se destacaram mais em um dos fragmentos, como Henriettea saldanhaei, Platymiscium floribundum e Tapirira guianensis, em ARI (Tab. 2) e Myrcia multiflora, Tabebuia umbellata e Inga laurina, em CM (Tab. 3).

As comunidades de árvores analisadas, conforme esperado, foram compostas principalmente por espécies peculiares de áreas alagadas. Das 31 espécies amostradas, quatro $(33,1 \%$ do total de indivíduos amostrados) foram peculiares exclusivas de áreas alagadas, sete $(57,9 \%)$ peculiares não exclusivas de áreas alagadas, nove $(2,3 \%)$ complementares de áreas secas e sete $(5,1 \%)$ complementares indiferentes. Quatro espécies $(1,6 \%)$ não foram classificadas: Andira anthelmia, Marlieria sylvatica, Myrcia hexasticha e Polygonaceae sp1 (Tab. 1).

As curvas de rarefação (Fig. 2) calculadas para riqueza e diversidade de espécies indicaram que os fragmentos estudados não diferem entre si (21 espécies, $\mathrm{H}^{\prime}=1,75$ nats.ind. ${ }^{-1}$ em ARI e 20 espécies, $H^{\prime}=1,99$ nats.ind.$^{-1} \mathrm{em} \mathrm{CM}$ ). Estes valores, entretanto, são baixos quando comparados com trechos de matas de solos mais drenados da região e que utilizaram mesmo critério mínimo de inclusão (DAP $\geq 10 \mathrm{~cm}$ ) (Tab. 4), conforme esperado. Os valores de diversidade de espécies nos dois fragmentos estão no limite inferior dos valores encontrados para outras matas sujeitas ao alagamento periódico no sudeste brasileiro que utilizaram critério mínimo de inclusão de 3,2 ou $5 \mathrm{~cm}$ de DAP (Tab. 4). Os dados de uniformidade (ARI $=0,57, \mathrm{CM}=0,66$ ) indicam a ocorrência de dominância de espécies nestes fragmentos. Esta dominância está representada principalmente pela alta abundância de Symphonia globulifera e Callophyllum brasiliense.

A floresta periodicamente inundada da Rebio apresentou maior similaridade florística, calculada pelo índice de Sørensen, com outras matas sujeitas ao alagamento periódico do solo, mesmo com aquelas geograficamente mais distantes, como as matas higrófilas do interior de São Paulo (Fig. 3). 


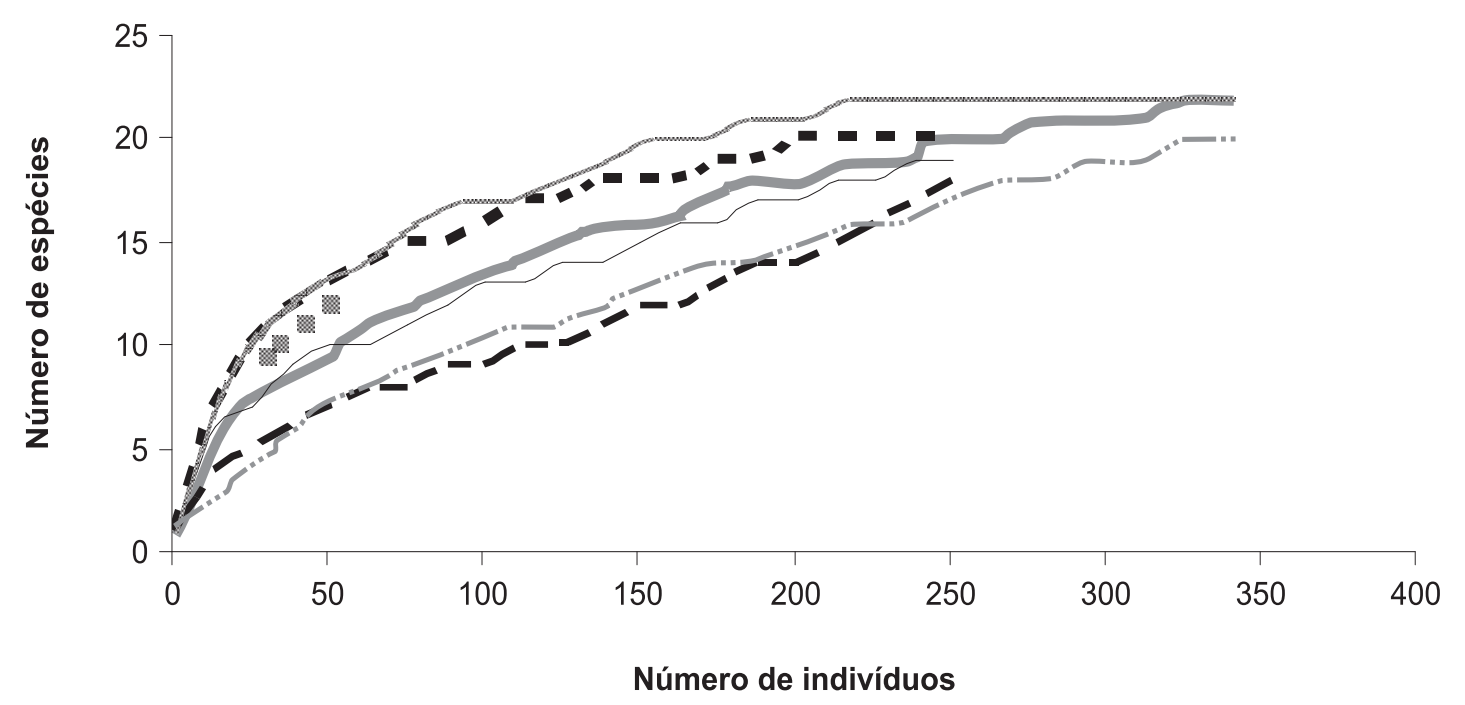

a

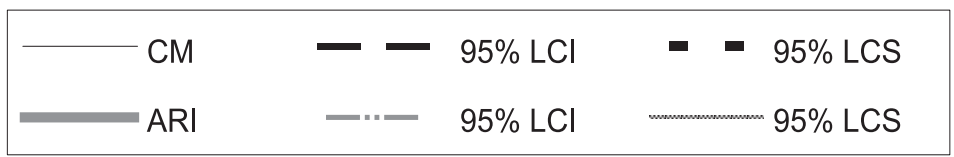

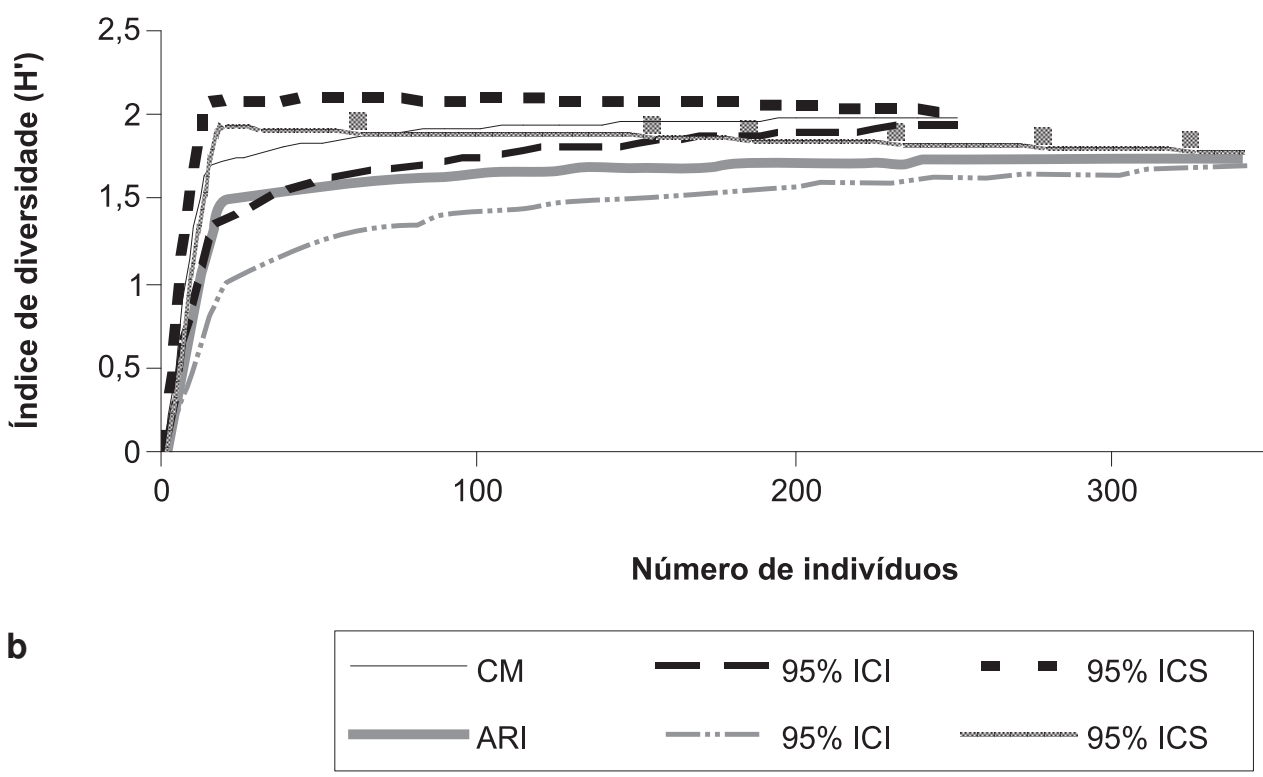

Figura 2 - Curvas de rarefação com valores medianos de riqueza (a) e diversidade (b) (H') de espécies e seus limites de confiança (95\%) inferiores (LCI) e superiores (LCS) para os dois fragmentos, riqueza (a) e diversidade (b), de floresta atlântica periodicamente alagada na Reserva Biológica de Poço das Antas, RJ. 
Tabela 2 - Parâmetros fitossociológicos das espécies arbóreas amostradas em 0,36 ha no fragmento de floresta de baixada periodicamente inundada ARI, na Reserva Biológica de Poço das Antas $\mathrm{RJ}$, ordenadas pelo $\mathrm{VC}$. $\mathrm{Ni}=$ número de indivíduos; $\mathrm{Ab}=$ área basal $\left(\mathrm{m}^{2}\right) ; \mathrm{DR}=$ densidade relativa $(\%) ; \mathrm{DoR}=$ dominância relativa $(\%) ; \mathrm{VC}=$ valor de cobertura.

\begin{tabular}{|c|c|c|c|c|c|c|}
\hline \multicolumn{2}{|c|}{ Espécie } & \multirow{2}{*}{$\begin{array}{l}\text { Ni } \\
181\end{array}$} & \multirow{2}{*}{$\begin{array}{l}\mathbf{A b} \\
4,87\end{array}$} & \multirow{2}{*}{$\begin{array}{l}\text { DR } \\
50,3\end{array}$} & \multirow{2}{*}{$\begin{array}{l}\text { DoR } \\
37,1\end{array}$} & \multirow{2}{*}{$\begin{array}{l}\text { VC } \\
87,4\end{array}$} \\
\hline 1 & Calophyllum brasiliense & & & & & \\
\hline 2 & Tabebuia cassinoides & 40 & 2,52 & 11,0 & 19,2 & 30,3 \\
\hline 3 & Symphonia globulifera & 54 & 1,90 & 14,9 & 14,5 & 29,4 \\
\hline 4 & Platymiscium floribundum & 10 & 1,48 & 2,76 & 11,3 & 14,0 \\
\hline 5 & Henriettea saldanhaei & 35 & 0,95 & 9,7 & 7,2 & 16,9 \\
\hline 6 & Tapirira guianensis & 9 & 0,29 & 2,5 & 2,2 & 4,7 \\
\hline 7 & Polygonaceae sp. & 2 & 0,19 & 0,5 & 1,4 & 2,0 \\
\hline 8 & Guapira hoenei & 3 & 0,15 & 0,8 & 1,1 & 2,0 \\
\hline 9 & Cordia sellowiana & 4 & 0,09 & 1,1 & 0,7 & 1,8 \\
\hline 10 & Pseudobombax grandiflorum & 3 & 0,10 & 0,8 & 0,8 & 1,6 \\
\hline 11 & Talauma ovata & 2 & 0,13 & 0,5 & 1,0 & 1,5 \\
\hline 12 & Tabebuia umbellata & 3 & 0,08 & 0,8 & 0,7 & 1,5 \\
\hline 13 & Eriotheca pentaphyla & 3 & 0,07 & 0,8 & 0,6 & 1,4 \\
\hline 14 & Euterpe edulis & 2 & 0,06 & 0,6 & 0,4 & 1,0 \\
\hline 15 & Syzigium jambos & 2 & 0,04 & 0,6 & 0,3 & 0,9 \\
\hline 16 & Tibouchina granulosa & 1 & 0,07 & 0,3 & 0,5 & 0,8 \\
\hline 17 & Pseudopiptadenia contorta & 2 & 0,02 & 0,5 & 0,2 & 0,7 \\
\hline 18 & Marlieria sylvatica & 2 & 0,02 & 0,5 & 0,2 & 0,7 \\
\hline 19 & Sarcaulus brasiliensis & 1 & 0,04 & 0,3 & 0,3 & 0,6 \\
\hline 20 & Alchornea triplinervia & 1 & 0,01 & 0,3 & 0,1 & 0,4 \\
\hline 21 & Myrsine coriacea & 1 & 0,01 & 0,3 & 0,1 & 0,4 \\
\hline
\end{tabular}

Tabela 3 - Parâmetros fitossociológicos das espécies arbóreas amostradas em 0,36 ha no fragmento de floresta de baixada periodicamente inundada CM, na Reserva Biológica de Poço das Antas $\mathrm{RJ}$, ordenadas pelo $\mathrm{VC} . \mathrm{Ni}=$ número de indivíduos; $\mathrm{Ab}=$ área basal $\left(\mathrm{m}^{2}\right) ; \mathrm{DR}=$ densidade relativa $(\%) ;$ DoR $=$ dominância relativa $(\%) ; \mathrm{VC}=$ valor de cobertura.

\begin{tabular}{|c|c|c|c|c|c|c|}
\hline \multicolumn{2}{|c|}{ Espécie } & \multirow{2}{*}{\begin{tabular}{|l}
$\mathbf{N i}$ \\
83
\end{tabular}} & \multirow{2}{*}{\begin{tabular}{|l|}
$\mathbf{A b}$ \\
5,48
\end{tabular}} & \multirow{2}{*}{\begin{tabular}{|l} 
DR \\
31,3
\end{tabular}} & \multirow{2}{*}{\begin{tabular}{|l|} 
DoR \\
41,1
\end{tabular}} & \multirow{2}{*}{\begin{tabular}{|l|} 
VC \\
72,4
\end{tabular}} \\
\hline 1 & Calophyllum brasiliense & & & & & \\
\hline 2 & Tabebuia cassinoides & 52 & 1,75 & 19,4 & 13,1 & 32,5 \\
\hline 3 & Myrcia multiflora & 37 & 2,14 & 13,8 & 16,1 & 29,9 \\
\hline 4 & Symphonia globulifera & 40 & 1,78 & 14,9 & 13,3 & 28,2 \\
\hline 5 & Inga laurina & 16 & 0,85 & 6,0 & 6,4 & 12,4 \\
\hline 6 & Tabebuia umbellata & 17 & 0,69 & 6,3 & 5,2 & 11,5 \\
\hline 7 & Polygonaceae sp. & 4 & 0,18 & 1,5 & 1,3 & 2,8 \\
\hline 8 & Cordia sellowiana & 5 & 0,06 & 1,9 & 0,5 & 2,4 \\
\hline 9 & Syzigium jambos & 1 & 0,10 & 0,4 & 0,8 & 1,2 \\
\hline 10 & Gomidesia fenzliana & 2 & 0,03 & 0,8 & 0,2 & 1,0 \\
\hline 11 & Inga striata & 1 & 0,06 & 0,4 & 0,5 & 0,9 \\
\hline 12 & Myrcia racemosa & 1 & 0,06 & 0,4 & 0,5 & 0,9 \\
\hline 13 & Eriotheca pentaphyla & 1 & 0,03 & 0,4 & 0,2 & 0,6 \\
\hline 14 & Pseudobombax grandiflorum & 1 & 0,02 & 0,4 & 0,2 & 0,6 \\
\hline 15 & Platymiscium floribundum & 1 & 0,02 & 0,4 & 0,2 & 0,6 \\
\hline 16 & Mimosa bimucronata & 1 & 0,02 & 0,4 & 0,1 & 0,5 \\
\hline 17 & Calyptrantes brasiliensis & 1 & 0,01 & 0,4 & 0,1 & 0,5 \\
\hline 18 & Andira onthelmia & 1 & 0,01 & 0,3 & 0,1 & 0,4 \\
\hline 19 & Myrcia hexasticha & 1 & 0,01 & 0,3 & 0,1 & 0,4 \\
\hline 20 & Inga edulis & 1 & 0,01 & 0,3 & 0,1 & 0,4 \\
\hline
\end{tabular}


Tabela 4 - Estrutura arbórea das duas florestas periodicamente inundadas da Reserva Biológica de Poço das Antas, RJ (ARI e CM) e comparação com outras florestas brasileiras. DAP = diâmetro a altura do peito da amostragem; $\mathrm{Ni}=$ número de indivíduos por hectare; $\mathrm{S}=$ número de espécies; $\mathrm{H}^{\prime}=$ índice de diversidade de Shannon (nats.ind. $\left.{ }^{-1}\right) ; \mathrm{AB}=$ área basal $\left(\mathrm{m}^{2} \mathrm{ha}^{-1}\right)$.

\begin{tabular}{|c|c|c|c|c|c|c|c|c|}
\hline Local & Tipo de floresta & Autor & DAP & Área & $\mathbf{N i}$ & $\mathbf{S}$ & $\mathbf{H}^{\prime}$ & $\mathbf{A B}$ \\
\hline $\begin{array}{l}\text { ARI (Poço } \\
\text { das Antas - RJ) }\end{array}$ & $\begin{array}{l}\text { Baixada periodicamente } \\
\text { alagada }\end{array}$ & Este trabalho & $\geq 10,0$ & 0,36 & 1003 & 21 & 1,75 & 36,5 \\
\hline $\begin{array}{l}\text { CM (Poço } \\
\text { das Antas - RJ) }\end{array}$ & $\begin{array}{l}\text { Baixada periodicamente } \\
\text { alagada }\end{array}$ & balho & $\geq 10,0$ & 0,36 & 2 & 20 & 1,99 & 37,0 \\
\hline Poço das Antas - RJ & Baixada aluvial & Guedes-Bruni (1998) & $\geq 10,0$ & 1,00 & 486 & 97 & 3,90 & 24,0 \\
\hline Poço das Antas - RJ & $\begin{array}{l}\text { Baixada permanentemente } \\
\text { alagada }\end{array}$ & Scarano et al. (1998) & $\geq 3,5$ & 0,50 & - & 59 & 1,30 & - \\
\hline Campinas - SP & Higrófila & Toniato et al. (1998) & $\geq 3,2$ & 0,20 & 4775 & 55 & 2,80 & 32,5 \\
\hline Campinas - SP & Higrófila & Torres et al. (1994) & $\geq 5,0$ & 0,87 & 1068 & 33 & 2,45 & - \\
\hline Itatinga - SP & Higrófila & Ivanauskas et al. (1997) & $\geq 5,0$ & 1,00 & 1242 & 39 & 2,75 & - \\
\hline Poconé - MT & Cambarazal & $\begin{array}{l}\text { Nascimento \& Cunha } \\
\text { (1989) }\end{array}$ & $\geq 3,2$ & 0,15 & 420 & 23 & 1,56 & - \\
\hline $\begin{array}{l}\text { São Franc. do } \\
\text { Itabapoana - RJ }\end{array}$ & Tabuleiro & $\begin{array}{l}\text { Silva \& Nascimento } \\
(2001)\end{array}$ & $\geq 10,0$ & 1,00 & 564 & 83 & 3,21 & 15,0 \\
\hline Poço das Antas - RJ & Submontana & Guedes-Bruni (1998) & $\geq 10,0$ & 1,00 & 564 & 169 & 4,50 & 25,0 \\
\hline União - RJ & Submontana & Rodrigues (2004) & $\geq 10,0$ & 1,20 & 734 & 250 & 4,90 & 32,0 \\
\hline Imbé - RJ & Submontana & Moreno et al. (2003) & $\geq 10,0$ & 0,60 & 767 & 125 & 4,21 & 41,9 \\
\hline Paraíso - RJ & Submontana & Kurtz \& Araujo (2000) & $\geq 5,0$ & - & 1370 & 138 & 4,20 & 57,2 \\
\hline
\end{tabular}

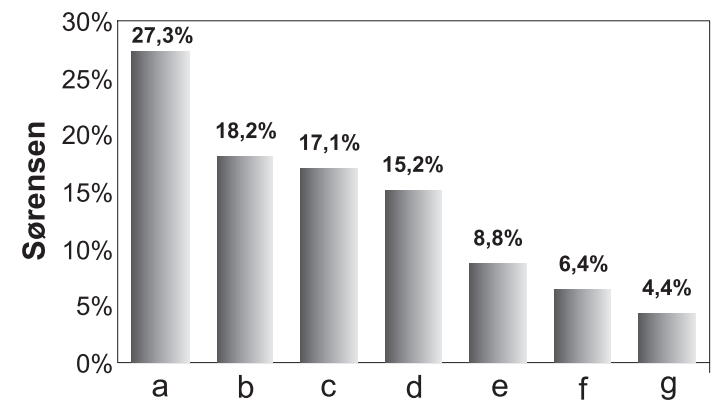

Figura 3 - Similaridade florística (índice de sørensen) entre a floresta periodicamente inundada da Reserva Biológica de Poço das Antas, RJ e alguns trechos de floresta atlântica com ou sem alagamento periódico, e florestas higrófilas de São Paulo, a partir do coeficiente de Sørensen. (a) floresta periodicamente alagada, Jurubatiba (Araujo et al. 1998); (b) floresta higrófila, Itatinga (Ivanauskas et al. 1997); (c) floresta higrófila, Campinas (Toniato et al. 1998); (d) floresta submontana, Reserva Biológica de Poço das Antas, Silva Jardim (Guedes-Bruni 1998); (e) floresta submontana, Silva Jardim (Borém \& Oliveira-Filho 2002); (f) floresta submontana, Reserva Biológica União, Rio das Ostras (Rodrigues 2004); (g) floresta submontana, Imbé, Campos dos Goytacazes (Moreno et al. 2003).

\section{Discussão}

Nos dois fragmentos estudados, Myrtaceae e Leguminosae apareceram como as famílias de maior riqueza, seguindo a tendência para floresta atlântica de Terras Baixas no Sudeste (Oliveira-Filho \& Fontes 2000). Normalmente, estas famílias encontram-se listadas entre as mais ricas nos levantamentos realizados na floresta atlântica do estado do Rio de Janeiro (Guedes-Bruni 1998, Neves 1999, Lima 2000, Silva \& Nascimento 2001, Kurtz \& Araujo 2000, Borém \& Ramos 2001, Borém \& Oliveira-Filho 2002, Rodrigues 2004, Carvalho et al. 2006).

A alta densidade de Calophyllum brasiliense, Symphonia globulifera e Tabebuia cassinoides nas florestas periodicamente alagadas estudadas e a ausência ou ocorrência em baixa densidade em florestas não inundáveis da região (Guedes-Bruni 1998, Neves 1999, Silva \& Nascimento 2001, Kurtz \& Araujo 2000, Borém \& Ramos 2001, Borém \& Oliveira-Filho 2002, Moreno et al. 2003, Rodrigues 2004, Carvalho et al. 2006), sugere a preferência destas espécies por habitat 
alagado. Áreas com alagamento e conseqüente saturação hídrica do solo geram condições de estresse para a maior parte das espécies vegetais terrestres (ver Crawford 1989). Por outro lado, algumas plantas que provavelmente têm sua história evolutiva relacionada a tais habitats se tornam características nestas comunidades (Gentry 1992). Portanto, assim como neste estudo, no sudeste brasileiro Tabebuia umbellata, Tapirira guianensis e Calophyllum brasiliense, sempre ocorreram com destaque nos levantamentos realizados em florestas higrófilas (Torres et al. 1994, Ivanauskas et al. 1997, Toniato et al. 1998). Desse modo, conforme sugerido por Joly (1991), a saturação hídrica do solo aparentemente funciona como o principal fator de seleção natural das espécies na formação de tais florestas em escala local e regional.

A ocorrência da grande maioria (ca. 90\%) das espécies amostradas como peculiar, exclusiva ou não, de áreas alagadas, também foi observado para as florestas higrófilas do interior de São Paulo (Torres et al. 1994, Ivanauskas et al. 1997, Toniato et al. 1998). As espécies consideradas peculiares exclusivas desse tipo de formação florestal foram Tabebuia cassinoides, Symphonia globulifera, Tabebuia umbellata e Talauma ovata, sendo as duas últimas citadas na literatura como típicas de floresta de brejo (Ivanauskas et al. 1997), e encontradas ocupando posições de destaque em outras florestas higrófilas no interior de São Paulo (Torres et al. 1994, Ivanauskas et al. 1997, Toniato et al. 1998). No estado do Rio de Janeiro, essas espécies aparentam ser exclusivas de florestas sujeitas ao alagamento do solo, aparecendo apenas em levantamentos florísticos de florestas de baixada periodicamente ou permanentemente alagada (Scarano et al. 1997, Scarano et al. 1998, Araujo et al. 1998, Lima et al. 2006), sendo ausentes ou raras em florestas com solos mais drenados (Guedes-Bruni 1998, Neves 1999, Silva \& Nascimento 2001, Kurtz \& Araujo 2000, Borém \& Ramos 2001, Borém \& Oliveira-Filho 2002, Moreno et al. 2003, Rodrigues 2004, Carvalho et al. 2006).
As espécies peculiares não exclusivas e, portanto, que mais contribuíram na composição florística dos fragmentos, são Calophyllum brasiliense, Henriettea saldanhaei, Platymiscium floribundum, Inga laurina, Myrcia multiflora, Alchornea triplinervia e Sarcaulus brasiliensis. Todas essas espécies já foram descritas em outras florestas inundáveis no estado do Rio de Janeiro (Scarano et al. 1997, Scarano et al. 1998, Araujo et al. 1998). Algumas dessas espécies, em especial Calophyllum brasiliense, foram registradas compondo o estrato arbóreo de florestas higrófilas no interior de São Paulo (Torres et al. 1994, Ivanauskas et al. 1997, Toniato et al. 1998), em florestas ciliares nos estados de Minas Gerais (Oliveira-Filho et al. 1994), São Paulo (Salis et al. 1994) e Paraná (Dias et al. 1998), e em florestas mais drenadas do Centro-Norte Fluminense (Neves 1999, Rodrigues 2004), neste último caso, entretanto, sem destaque nos parâmetros fitossociológicos.

A presença de espécies normalmente relacionadas a florestas com solos bem drenados no Centro-Norte Fluminense (Neves 1999, Silva \& Nascimento 2001, Borém \& Ramos 2001, Borém \& Oliveira-Filho 2002, Rodrigues 2004, Carvalho et al. 2006) tais como Inga striata, Mimosa bimucronata, Pseudopiptadenia contorta, Tibouchina granulosa, Myrsine coriacea, Myrcia racemosa e Guapira hoenei nos fragmentos estudados sugerem uma alta valência ecológica destas espécies complementares de áreas secas. Tibouchina granulosa, por exemplo, é uma espécie muito comum na Floresta Ombrófila Densa do estado do Rio de Janeiro, sendo observada principalmente em formações secundárias como capoeiras e capoeirões, e nas bordas de diversos fragmentos florestais, principalmente em solos bem drenados (Lorenzi 1992). Em outro exemplo, Pseudopiptadenia contorta aparece como uma das espécies de maior VI (valor de importância) numa floresta de baixada de solos bem drenados a menos de $20 \mathrm{~km}$ dos fragmentos estudados (Borém \& Oliveira-Filho 
2002) e para uma Floresta Estacional Semidecidual no Norte Fluminense (Silva \& Nascimento 2001).

As espécies complementares indiferentes Tapirira guianensis, Pseudobombax grandiflorum, Eriotheca pentaphylla, Euterpe edulis, Cordia sellowiana, Inga edulis e a exótica Syzygium jambos, que, apesar de estarem comumente descritas para florestas sob influência do alagamento periódico do solo (Torres et al. 1994, Oliveira-Filho et al. 1994, Ivanauskas et al. 1997, Toniato et al. 1998, Van Den Berg \& Oliveira-Filho 2000, Carvalho et al. 2000), também podem ser encontradas em diversos remanescentes de solos bem drenados no estado do Rio de Janeiro (Guedes-Bruni 1998, Neves 1999, Kurtz \& Araujo 2000, Silva \& Nascimento 2001, Moreno et al. 2003, Rodrigues 2004, Carvalho et al. 2006). De fato, Oliveira-Filho \& Fontes (2000) citaram Eriotheca pentaphylla, Euterpe edulis e Inga edulis como espécies de ampla ocorrência nas florestas de terras baixas do sudeste brasileiro. Entretanto, Tapirira guianensis e Cordia sellowiana são citadas como supertramps, ou seja, espécies com ampla distribuição e ocorrência em grande parte do território nacional.

Os valores de densidade e de área basal por hectare dos fragmentos estudados estão entre os mais altos quando comparados com outros trechos preservados de floresta atlântica no estado do Rio de Janeiro (Tab. 4) e em muitos casos esta diferença seria mais evidente se os critérios de inclusão amostral fossem semelhantes (DAP $\geq 10 \mathrm{~cm}$ ). Aliado a estas características, observações de campo tais como o grande número de árvores de grande porte, a alta concentração e riqueza de epífitas nos diferentes estratos das florestas, além da ausência de vestígios de árvores cortadas indicam, segundo Clark (1996), que os fragmentos estudados são maduros.

De maneira geral, florestas sujeitas ao alagamento tendem a apresentar menor diversidade de espécies em relação aquelas com solos mais drenados, pois são poucas as espécies que desenvolveram adaptações que possibilitem sua sobrevivência nesses ambientes (Joly 1991). Os índices de Shannon (H') para os fragmentos estudados foram considerados baixos em relação a outros levantamentos em trechos de solos mais drenados de floresta atlântica ( $H^{\prime}$ entre 3,21 e 4,93 nats.ind. ${ }^{-1}$ ), e no limite inferior aos obtidos para outras florestas higrófilas (brejo) ou com alagamento quase que permanente do solo (e.g. cambarazal e floresta de baixada permanentemente alagada) (Tab. 4). Scarano et al. (1998), ao levantarem um trecho de floresta de baixada permanentemente inundada dentro da Rebio, muito próximo aos fragmentos estudados e com dominância de Tabebuia cassinoides, encontraram um valor de diversidade de Shannon ainda menor, de 1,30 nats.ind.$^{-1}$. Isto indica que ambientes com estresse hídrico tendem a possuir baixa diversidade de espécies (Nascimento \& Cunha 1989, Joly 1991, Ivanauskas et al. 1997, Toniato et al. 1998), sendo o alagamento do solo o fator restritivo, onde quanto maior o período de encharcamento do solo, menor a diversidade encontrada.

A maior similaridade de espécies observada entre a floresta periodicamente alagada de Rebio com aquelas sujeitas ao alagamento do solo (Fig. 3), mesmo as mais distantes, indicam a preferência das espécies por áreas sujeitas ao alagamento do solo. Estes resultados confirmam que as florestas periodicamente inundadas amostradas têm a sua florística constituída em parte pelas formações florestais adjacentes, através da presença de espécies complementares de áreas secas, todavia com uma presença marcante de espécies adaptadas ao alagamento e peculiares a esses ambientes.

Após as obras de drenagem, promovidas pelo Departamento Nacional de Obras e Saneamento, nas décadas de 50 a 70, as florestas de baixada inundáveis da região Centro-Norte Fluminense foram praticamente extintas, restando poucos remanescentes (Castro \& Fernandez 2002). Apesar do histórico de perturbação relativamente recente (ca. 35 anos), os dois fragmentos estudados 
encontram-se sem registros de perturbações antrópicas desde então, apresentando aspectos florísticos e estruturais de florestas bem conservadas. Por constituírem unidades fitogeográficas de características próprias e de extrema importância para a manutenção da fauna associada e dos recursos hídricos naturais, sugerimos que as florestas de baixada inundável sejam prioritárias em programas de conservação a serem desenvolvidos na região da bacia hidrográfica do rio São João.

\section{Agradecimentos}

Aos pesquisadores Carlos R. RuizMiranda, Dora M. Villela, Jorge Assumpção e Josival S. Souza pelas críticas ao manuscrito; a AMLD pelo apoio logístico. Ao IBAMA, por permitir o acesso à área de estudo; ao técnico de campo Maurício Pacheco, e aos biólogos Guilherme R. Rabelo, Guilherme V. Faria, Glauce M. Gonçalves e Diogo O. Santos, pelo auxílio nas atividades de campo; ao PROBIO/ PRONABIO/BIRD/MMA pelo apoio financeiro e ao CNPq pela bolsa de I.C. (PIBIC) concedida ao primeiro autor no período de 1999 a 2001.

\section{REFERÊNCIAS BIBLIOGRÁFICAS}

Araujo, D. S. D.; Scarano, F. R.; Sa, C. F. C.; Kurtz, B. C.; Zaluar, H. L. T.; Montezuma, R. C. M. \& Oliveira, R. C. 1998. Comunidades vegetais do Parque Nacional da restinga de Jurubatiba. In Esteves, F. A. (ed.). Ecologia das Lagoas Costeiras do Parque Nacional de Jurubatiba e do Município de Macaé. NUPEM/UFRJ. Pp. 39-62.

Aselmann, I. \& Crutzen, P. J. 1989. Global distribution of natural freshwater wetlands and rice paddies, their net primary productivity, seasonality and possible methane emission. Journal of Atmospheric Chemistry 8: 307-358.

Borém, R. A. T. \& Oliveira-Filho, A. T. 2002. Fitossociologia do estrato arbóreo em uma topossequência alterada de Mata Atlântica, no município de Silva JardimRJ, Brasil. Revista Árvore 26: 727-742.
\& Ramos, D. P. 2001. Estrutura fitosso-ciológica da comunidade arbórea de uma topossequência pouco alterada de uma área de floresta atlântica no município de Silva Jardim-RJ. Revista Árvore 25: 131-140.

Brower, J. E. \& Zar, J. H. 1984. Field and laboratory methods for general ecology. 2nd ed., W. C. Brown Company Publishers, Iowa, 226p.

Câmara, I. G. 1991. Plano de ação para a Mata Atlântica. Fundação SOS Mata Atlântica, São Paulo, 152p.

Carvalho, D. A.; Oliveira-Filho, A. T.; Vilela, E. A. \& Curi, N. 2000. Florística e estrutura da vegetação arbórea de um fragmento de floresta semidecidual às margens do reservatório da usina hidrelétrica Dona Rita (Itambé do Mato Dentro, MG). Acta Botanica Brasilica 14: 37-55.

Carvalho, F. A.; Nascimento, M. T. \& Braga, J. M. A. 2006. Composição e riqueza florística do componente arbóreo da floresta atlântica submontana na região de Imbaú, município de Silva Jardim, RJ. Acta Botanica Brasilica 20(3): 741-754.

Castro, E. B. V. \& Fernandez, F. A. S. 2002. A fragmentação florestal na Reserva Biológica de Poço das Antas como conseqüência das intervenções de engenharia na bacia do Rio São João (RJ). In: Anais do III Congresso Brasileiro de Unidades de Conservação. Fundação O Boticário de Proteção à Natureza, Fortaleza. Pp. 649-659.

Clark, D. B. 1996. Abolishing virginity. Journal of Tropical Ecology 12: 735-739.

Constantino, R.; Britez, R. M.; Cerqueira, R.; Espindola, E. L. G.; Grelle, C. E. V.; Lopes, A. T. L.; Nascimento, M. T.; Rocha, O.; Rodrigues, A. A. F.; Scariot, A.; Sevilha, A. C. \& Tiepolo, G. 2005. Causas naturais da Fragmentação. In: D. Rambaldi, D. \& Oliveira, D. A. S. (orgs.). Efeitos da Fragmentação sobre a Biodiversidade: Recomendações de Políticas Públicas. $2^{\mathrm{a}}$ ed., MMA, Brasília. Pp. 43-63. 
Crawford, R.M. 1989. Studies in plant survival. Blackwell Scientific Publications, Oxford, 496p.

Cronquist, A. 1981. An integrated system of classification of flowering plants. Columbia University Press, New York, 1262p.

Dias, M. C.; Vieira, A. O. S.; Nakajima, J. N.; Pimenta, A. P. \& Lobo, P. C. 1998. Composição florística e fitossociologia do componente arbóreo das florestas ciliares do rio Igapó, na bacia do rio Tibagi, Tibagi, PR. Revista Brasileira de Botânica 21: 183-195.

Esteves, F. A. 1998. Considerations on the ecology of wetlands, with enphasis on brazilian floodplains ecosystems. In: Scarano, F. R. \& Franco, A. C. (eds.). Ecophysiological strategies of xerophytic and amphibious plants in the neotropics. Oecologia brasiliensis, IV, PPGE-UFRJ, Rio de Janeiro. Pp. 111-135.

Felfili, J. M. 1993.Structure and dynamics of a gallery forest in Central Brazil. PhD Thesis, Oxford University, Oxford, 180p.

Fundação SOS Mata Atlântica. 2002. Atlas da evolução dos remanescentes florestais e ecossistemas associados no domínio da Mata Atlântica no período 1995-2000. SOS Mata Atlântica/INPE/ISA, São Paulo. http.//w.w.w.sosmataatlantica.org.br.

Gentry, A. H. 1992. Bignoniaceae. Part II (Tribe Tecomeae). Flora Neotropica Monograph 25: 1-370.

Guedes-Bruni, R. R. 1998. Composição, estrutura e similaridade de dossel em seis unidades fisionômicas de Mata Atlântica no Rio de Janeiro. Tese de Doutorado. Universidade de São Paulo, São Paulo, 347p.

Gotelli, N. J. \& Entsminger, G. L. 2001. EcoSim: Null models software for ecology. Version 7.0. Acquired Intelligence Inc. \& KeseyBear. http://homepages.together.net/ qgentsmin/ ecosim.htm.

IBDF. 1981. Plano de Manejo da Reserva Biológica de Poço das Antas. Documento técnico, no 10, Ministério da Agricultura, Brasília.
Ivanauskas, N. M.; Rodrigues, R. R. \& Nave, A. G. 1997. Aspectos ecológicos de um trecho de floresta de brejo em Itatinga, SP: florística, fitossociologia e seletividade de espécies. Revista Brasileira de Botânica 20: 139-153.

Joly, C. A. 1991. Flooding tolerance in tropical trees. In: Jacksons, M. B.; Davis, D. D. \& Lambers, H. (eds.). Plant life under oxygen deprivation. SPB Academic Publishing, The Hague. Pp. 23-43. \& Crawford, R. M. M. 1982. Variation in tolerance and metabolic response to flooding in some tropical trees. Journal of Experimental Botany 33: 799-809.

Junk, W. J. 1989. Flood tolerance and tree distribution in Central Amazonian floodplains. In: Holm-Nielsen, L. B.; Nielsen, I. \& Baslev, H. (eds.). Tropical Forests. Academic Press, New York. Pp. 65-42.

Kubitzky, K. 1989. The ecogeographical differentiation of Amazonian inundation forests. Plant Systematics and Evolution 162: 285-304.

Kurtz, B. C. \& Araujo, D. S. D. 2000. Composição florística e estrutura do componente arbóreo de um trecho de Mata Atlântica na estação Estadual do Paraíso, Cachoeiras de Macacu, Rio de Janeiro, Brasil. Rodriguésia 71: 69-111.

Leitão-Filho, H. F. 1982. Aspectos taxonômicos das florestas do estado de São Paulo. Silvicultura em São Paulo 16: 197-206.

Lima, H. C. 2000. Leguminosas arbóreas da Mata Atlântica. Tese de Doutorado. Universidade Federal do Rio de Janeiro, Rio de Janeiro, 141p.

Lima, H. C.; Pessoa, S. V. A.; Guedes-Bruni, R.; Moraes, L. F. D.; Granzotto, S. V.; Iwamoto, S. \& Ciero, J. Di. (2006). Vegetação da Reserva Biológica de Poço das Antas, Mun. Silva Jardim, RJ, Brasil. Rodriguésia 57(3): 369-389.

Lorenzi, H. 1992. Árvores Brasileiras. Manual de identificação e cultivo de plantas arbóreas do Brasil. Editora Plantarum, Nova Odessa, São Paulo, 352p. 
Loureiro, M. F.; Faria, S. M.; James, E. K.; Pott, A. \& Franco, A. A. 1994. Nitrogenfixing stem nodules of the legume, Discolobium pulchellum Benth. New Phytologist 128: 283-295.

Maltby, E. 1991. Wetlands - Their status and role in the biosphere. In: Jacksons, M. B.; Davis, D. D. \& Lambers, H. (eds.). Plant life under oxygen deprivation. The Hague, SPB Academic Publishing. Pp. 3-21.

Medri, M. E.; Bianchini, E.; Pimenta, J. A.; Colli, S. \& Müller, C. 2002. Estudos sobre tolerância ao alagamento em espécies arbóreas nativas da bacia do rio Tibagi. In: Medri, M. E.; Bianchini, E.; Shibatta, O. A. \& Pimenta, J. A. (eds.). A bacia do rio Tibagi, Londrina. Pp. 133-172.

Milaré, E. 1991. Legislação ambiental no Brasil. Edições APMP. Série Cadernos informativos

Moraes, E. 2000. Estrutura populacional de Symphonia globulifera (Clusiaceae) em fragmentos de mata alagada nas Reservas Biológicas de Poço das Antas e União, RJ. Dissertação de Mestrado. Universidade Estadual do Norte Fluminense, Campos dos Goytacazes, 67p.

Moreno, M. R.; Nascimento, M. T. \& Kurtz, B. C. 2003. Estrutura e composição florística do estrato arbóreo em duas zonas altitudinais na Mata Atlântica de encosta da região do Imbé, RJ. Acta Botanica Brasilica 17: 371-386.

Mueller-Dumbois, D \& Ellenberg, H. 1974. Aims and methods of vegetation ecology. John \& Wiley Sons, New York, 347p.

Naiman, R. J. \& Décamps, H. 1997. The ecology of interfaces: riparian zones. Annual Review of Ecology and Systematics 28: 621-658.

Nascimento, M. T. \& Cunha, C. N. 1989. Estrutura e composição florística de um cambarazal no Pantanal de Poconé-MT. Acta Botanica Brasilica 3: 3-23.

Neves, G. M. S. 1999. Florística e estrutura da comunidade arbustivo-arbórea em dois remanescentes de floresta atlântica secundária - Reserva Biológica de Poço das Antas, Silva Jardim, RJ. Dissertação de Mestrado. Universidade Federal do Rio de Janeiro, Rio de Janeiro, 115p.

Oliveira-Filho, A. T. 1989. Composição florística e estrutura comunitária da floresta de galeria do córrego da Paciência, Cuiabá (MT). Acta Botanica Brasilica 3: 91-112.

Almeida, R. G.; Mello, J. M. \& Gavilanes, M. L. 1994. Estrutura fitossociológica e variáveis ambientais em um trecho da mata ciliar do córrego dos Vilas Boas, Reserva Biológica de Poço Bonito, Lavras (MG). Revista Brasileira de Botanica 17: 67-85.

\& Fontes, M. A. L. 2000. Patterns of floristic differentiation among Atlantic Forests in Southeastern Brazil and the influence of climate. Biotropica 32: 793-810.

Polhill, R. M. \& Raven, P. H. 1981. Advances in legumes systematics. Part 1. Royal Botanic Garden, Kew, 425p.

Prance, G. T. 1979. Notes on the vegetation of Amazon III. The terminology of Amazonian forest types subject to inundation. Brittonia 31: 26-38.

\& Schaller, G. B. 1982. Preliminary study of some vegetation types of the pantanal, Mato Grosso, Brazil. Brittonia 34: 228-251.

Programa Mata Atlântica 2004. Relatório Técnico-Científico. Rio de Janeiro. Jardim Botânico do Rio de Janeiro.

RADAMBRASIL. 1983. Levantamento de recursos naturais. Ministério das Minas e Energia, v.32, Rio de Janeiro, 768p.

Ratter, J. A.; Pott, A.; Pott, V. J.; Cunha, C. N. \& Haridasan, M. 1998. Observations on woody vegetation types in the pantanal and at Corumbá, Brazil. Revista Brasileira de Geografia 45: 503-525.

Rodrigues, P. J. F. P. 2004. A vegetação da Reserva Biológica União e os efeitos de borda na Mata Atlântica fragmentada. Tese de Doutorado. Universidade Estadual do Norte Fluminense, Campos dos Goytacazes, 153p. 
Salis, S. M.; Tamashiro, J. Y. \& Joly, C. A. 1994. Florística e fitossociologia do estrato arbóreo de um remanescente de mata ciliar do rio Jacaré-Pepira, Brotas, SP. Revista Brasileira de Botânica 17: 93-139.

Scarano, F. R.; Cattânio, J. H. \& Crawford, R. M. M. 1994. Root carbohydrate storage in young saplings of an amazonian tidal varzea forest before the onset of the wet season. Acta Botanica Brasilica 8: 129-139.

; Ribeiro, K. T.; Moraes, L. F. D. \& Lima, H. C. 1997. Plant establishment on flooded and unflooded patches of a freshwater swamp forest in southeastern Brazil. Journal of Tropical Ecology 14: 793-803.

; Rios, R. I. \& Esteves, F. A. 1998. Tree species richness, diversity and flooding regime: case studies of recuperation after anthropic impact in brazilian flood-prone forests. International Journal of Ecological and Environmental Sciences 24: 223-235.

Schluter, U. B.; Fürch, B. \& Joly, C. A. 1993. Physiological and anatomical adaptations by young Astrocariym jauari Mart. (Arecaceae) in periodically inundated biotopes of Central Amazonia. Biotropica 25: 384-396.

Shepherd, G. J. 1994. FITOPAC 1: Manual do usuário. Departamento de Botânica. Universidade Estadual de Campinas, Campinas, 59p.

Silva, G. C. \& Nascimento, M. T. 2001. Fitossociologia de um remanescente de mata sobre tabuleiros no norte do estado do Rio de Janeiro (Mata do Carvão). Revista Brasileira de Botânica 24: 51-62.

Takizawa, F. H. 1995. Levantamento pedológico e zoneamento ambiental na Reserva Biológica de Poço das Antas. Relatório Técnico, Departamento de Ciência do Solo, USP/ESALQ, Piracicaba.

Toniato, M. T. Z.; Leitão-Filho, H. F. \& Rodrigues, R. R. 1998. Fitossociologia de um remanescente de floresta higrófila (mata de brejo) em Campinas, SP. Revista Brasileira de Botânica 21: 197-210.

Torres, R. B.; Matthes, L. A. F. \& Rodrigues, R.R. 1994. Florística e estrutura do componente arbóreo de mata de brejo em Campinas, SP. Revista Brasileira de Botânica 17: 189-194.

van den Berg, E. \& Oliveira-Filho, A. T. 2000. Composição florística e estrutura fitossociológica de uma floresta ripária em Itutinga, MG, e comparação com outras áreas. Revista Brasileira de Botânica 23: 231-253.

Veloso, H. P.; Rangel Filho, A. L. R. \& Lima, J. C. A. 1991. Classificação da Vegetação Brasileira, adaptada a um sistema universal. IBGE, Rio de Janeiro, 123p.

Worbes, M. 1985. Structural and other adaptations to long-term flooding by trees in Central Amazonia. Amazoniana 9: 459-484.

World Conservation Monitoring Centre. 1992. Global biodiversity - status of the earth living resources. Chapman \& Hall, London, 614p. 